\title{
ESTUDO ELETROQUÍMICO SOBRE A OXIDAÇÃO DE BIODIESEL*
}

\author{
ELECTROCHEMICAL STUDY ABOUT THE OXIDATION OF BIODIESEL
}

\author{
ESTUDIO ELECTROQUÍMICO SOBRE LA OXIDACIÓN DE BIODIESEL
}

\author{
Isabelle Moraes Amorim Viegas \\ Verônica Diniz da Silva \\ Aldaléa Lopes Brandes Marques \\ Edmar Pereira Marques
}

\begin{abstract}
Resumo: Objetivando a preservação ambiental e a melhoria do desempenho dos motores dos transportes, a Agência Nacional do Petróleo, Gás Natural e Biocombustíveis estabelece a adição obrigatória de biodiesel ao diesel mineral em uma proporção de $5 \%$ de biodiesel, em volume, ao diesel. Um dos parâmetros de qualidade é o período de indução, que está relacionado com a estabilidade oxidativa do biodiesel e é determinado pelo método Rancimat. A RANP 14/12 estabelece o período de indução mínimo de 6 h, que indica que o biocombustível será estável por até 6 meses de armazenamento. O presente trabalho propõe uma metodologia eletroquímica para estudar o comportamento oxidativo do biodiesel, utilizando a técnica voltametria cíclica. O biodiesel metílico de soja, sintetizado a partir da rota com catálise básica homogênea, apresentou período de indução $4,88 \mathrm{~h}$ e foi utilizado em todos os experimentos deste trabalho. Dentre os meios estudados para os ensaios voltamétricos, optou-se pelo meio orgânico, utilizando ácido nítrico como eletrólito de suporte. A metodologia proposta apresentou boa resposta à oxidação eletroquímica do biodiesel, devido à boa linearidade de crescimento da corrente de pico anódico com as adições da amostra, com coeficiente de correlação 0,996, para concentrações de até 10,66 mg.mL-1 de biodiesel. A partir da concentração de 12,44 mg.mL-1, observa-se uma pequena perda de linearidade, que pode ser atribuída à limitação do processo catalítico na superfície do eletrodo de ouro e à possível formação de um filme de poliacetonitrila. Os resultados obtidos no presente trabalho confirmam a viabilidade do método proposto para o estudo eletroquímico sobre a oxidação do biodiesel.
\end{abstract}

Palavras-chave: Biodiesel. Estabilidade Oxidativa. Voltametria Cíclica.

Abstract: Aiming environmental preservation and improvement on performance of engines transports, the Brazilian National Agency of Petroleum, Natural Gas and Biofuels establishes the mandatory addition of biodiesel to mineral diesel in Brazil, in a proportion of $5 \%$ of biodiesel, in volume, to diesel. One of the quality parameters of the biodiesel is the induction period, which is related to its oxidative stability, it being determined by the Rancimat method. The RANP no 14/2012 establishes a minimum value of $6 \mathrm{~h}$ for the induction period, which indicates that the biofuel will be stable for 6 months storage. This work proposes an electrochemical methodology to study oxidative behavior of biodiesel, using cyclic voltammetry technique. A methylic soybean biodiesel was synthesized through a basic catalysis homogeneous route, presenting an induction period of $4,88 \mathrm{~h}$, which was utilized in all experiments of this work. Among all conditions evaluated for the voltammetric study, the organic middle was the chosen, using nitric acid as supporting electrolyte, because it presented the best voltammetric results, in terms of resolution and sensitivity. The proposed methodology presented a good response for the electrochemical oxidation study of biodiesel, due to the good linear relationship obtained between the anodic peak current and biodiesel concentration, with a correlation coefficient of 0,996 , to concentrations until 10,66 mg.mL-1 of biodiesel. From concentration of $12,44 \mathrm{mg} \cdot \mathrm{mL}-1$, a small deviation from linearity is observed, which could be attributed to the limitation of the catalytic process on the gold electrode surface and to the possible formation of a polyacetonitrile film. The results obtained in the present work confirm the viability of the proposed method to the electrochemical study about oxidation of biodiesel.

Keywords: Biodiesel. Oxidative Stability. Cyclic Voltammetry.

Resumen: Con el objetivo de preservar el medio ambiente y mejorar el desempeño de los motores de los transportes, la Agencia Nacional de Petróleo, Gas Natural y Biocombustibles establece la adición obligatoria de biodiesel al diesel mineral en una proporción de 5\% de biodiesel, en volumen, de diesel. Uno de los parámetros de calidad es el período de inducción, que tiene relación con la estabilidad oxidativa del biodiesel y se determina por el método Rancimat. La RANP 14/12 establece el período de inducción mínimo de $6 \mathrm{~h}$, que indica que el biocombustible será estable durante 6 meses de almacenamiento. En este trabajo se propone una metodología electroquímica para estudiar el comportamiento electroquímico de la oxidación de biodiesel, utilizando la técnica de voltametría cíclica. El biodiesel de soja metílico, sintetizado a partir de la ruta con catálisis básica homogénea, presentó período de inducción de 4,88 h y se utilizó en todos los experimentos de este trabajo. De las condiciones estudiadas para los ensayos de voltametría, el medio

Trabalho premiado durante o XXIV Encontro do SEMIC, realizado na UFMA entre os dias 05 a 08 de novembro de 2012.

*Artigo recebido em dezembro 2012

Aprovado em fevereiro 2013 
orgánico fue el elegido, usando ácido nítrico como electrólito de soporte. La metodología propuesta presenta una buena respuesta a la oxidación electroquímica del biodiesel, debido a la buena linealidad de aumento de la corriente de pico anódico con adición de muestra, con un coeficiente de correlación 0,996 para concentraciones de hasta 10,66 mg.mL-1 de biodiesel. Después de la concentración de $12,44 \mathrm{mg} . \mathrm{mL}-1$, una pequeña desviación de la linealidad se observa, lo que podría atribuirse a la limitación del proceso catalítico en la superficie del electrodo de oro y a la posible formación de una película de poliacetonitrila. Los resultados obtenidos en el presente trabajo confirman la viabilidad del método propuesto para el estudio sobre la oxidación electroquímica de biodiesel.

Palabras clave: Biodiesel. Estabilidad oxidativa. Voltametría cíclica.

\section{INTRODUÇÃO}

A matriz energética mundial tem sido muito debatida a fim de buscar novos combustíveis que se tornem gradualmente substitutos dos derivados de petróleo, uma vez que as reservas de combustíveis fósseis estão se tornando cada vez mais limitadas e futuramente esgotáveis (SCHUCHARDT; SERCHELI; VARGAS, 1998). Nas últimas décadas, a busca por combustíveis alternativos tem sido motivada também pelas mudanças climáticas globais, causadas principalmente pela emissão de gases que provocam o efeito estufa (COLARES, 2008), (MITCHELL; JOHNS, 1997).

Nesse contexto, os biocombustíveis se evidenciam como principais substituintes oriundos da biomassa para alimentar os motores dos transportes, por serem renováveis, biodegradáveis e ambientalmente corretos. Biocombustível é uma fonte de energia produzida da biomassa, que pode ser sólida, líquida ou gasosa. Os mais comuns são o etanol e o biodiesel, utilizados para suprir, respectivamente, a gasolina e o óleo diesel (DERMIBAS, 2009). Atualmente o etanol é o biocombustível mais usado no mundo todo, e a matéria-prima para a produção etílica depende das condições da agricultura local (DERMIBAS, 2009).

Biodiesel é um combustível sintético produzido tipicamente de óleos vegetais ou gorduras animais. Pode ser misturado ao diesel de petróleo ou utilizado puro, neste último caso precisando de algumas adaptações no motor (DERMIBAS, 2009). A partir de janeiro de 2010, a Agência Nacional do Petróleo, Gás Natural e Biocombustíveis (ANP) instituiu a adição obrigatória de biodiesel ao diesel mineral em uma proporção de $5 \%$ de biodiesel, em volume (B5) (AGÊNCIA NACIONAL DO PETRÓLEO, GÁS NATURAL E BIOCOMBUSTÍVEIS, 2012a ).

Segundo dados da ANP, em 2011, a principal matéria-prima do biodiesel, no Brasil, foi o óleo de soja, representando $81,2 \%$ da produção total (AGÊNCIA NACIONAL DO PETRÓLEO, GÁS NATURAL E BIOCOMBUSTÍVEIS, 2012a). Mundialmente, o óleo de soja também repre senta a maior parte da produção (FERRARI; OLIVEIRA; SCABIO, 2005), porém há mais de 300 matérias-primas que podem ser utilizadas para tal finalidade (SHAHID; JAMAL, 2011). O óleo de soja apresenta boas propriedades, além de que a cultura da soja já é bem implementada em diversos países. Em contraposição, tem-se a sua principal desvantagem: a competição entre os setores alimentício e energético, ambos dependentes dessa oleaginosa comestível, o que acaba por gerar muitas discussões éticas e políticas, provocando grande flutuação do preço da soja (DERMIBAS, 2009), (BALAT, 2011).

\subsection{Biodiesel de soja: produção e propriedades}

O biodiesel é composto de mono-alquil ésteres de ácidos graxos derivado de fontes renováveis, produzido principalmente a partir de uma reação de transesterificação de triglicerídeos com álcool, na presença de um catalisador. A reação pode ser esquematizada pela Figura 1. Como subprodutos obtêm-se glicerol livre e, em menor quantidade, sabões residuais, glicerídeos parciais e resíduos de catalisador e álcool (FERRARI; OLIVEIRA; SCABIO, 2005).

Figura 1 - Reação de transesterificação de triglicerídeos com álcool

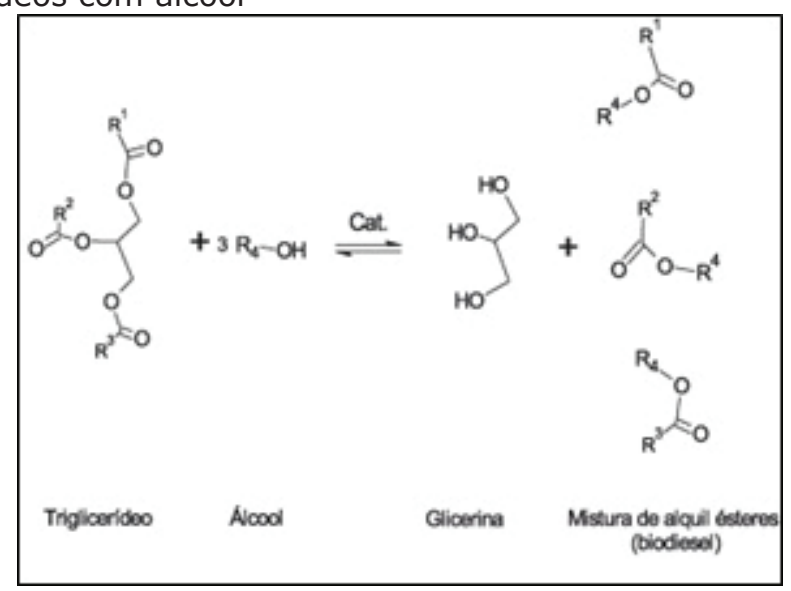

Fonte: Lôbo, Ferreira e Da Cruz (2009)

Os catalisadores mais comuns são ácidos, hidróxidos, alcóxidos e carbonatos de metais alcalinos, enzimas e bases não iônicas. Os hidróxidos são mais empregados, principalmente hidróxido de sódio e de potássio, pois, além de acelerarem a reação, proporcionam maior rendimento (SCHUCHARDT; SERCHELI; VARGAS, 1998), (FERRARI; OLIVEIRA; SCABIO, 2005).

Os álcoois mais utilizados são os de cadeia curta, como metanol, etanol, propanol e butanol, sendo os dois primeiros mais vantajosos por reduzirem o tempo reacional. 
Para alcançar um bom rendimento e favorecer a separação de fases entre o biodiesel e os subprodutos, é fundamental que se utilize excesso do álcool, devido ao caráter reversível da reação de transesterificação. Além disso, o óleo vegetal deve possuir baixo teor de ácidos graxos livres (baixo índice de acidez), pois estes reagem com a base (catalisador), formando sabões e reduzindo o rendimento da reação (FERRARI; OLIVEIRA; SCABIO, 2005), (SCHUCHARDT; SERCHELI; VARGAS, 1998).

As propriedades físico-químicas do biodiesel precisam ser bastante semelhantes às do diesel mineral, pois é isto que possibilita a adição do biocombustível ao diesel (FERRARI; OLIVEIRA; SCABIO, 2005). Na Tabela 1, estão especificadas as propriedades dos biodieseis metílico e etílico de soja sintetizados e caracterizados por Candeia (CANDEIA, 2008).
Na Tabela 2, segue a composição graxa do óleo de soja (AGÊNCIA NACIONAL DE VIGILÂNCIA SANITÁRIA, 2000).

Tabela 2 - Composição dos ésteres de ácidos graxos do óleo de soja

\begin{tabular}{c|c|c}
\hline $\begin{array}{c}\text { No de carbonos e } \\
\text { insaturações }\end{array}$ & Ácido graxo & $\begin{array}{c}\text { Percentual } \\
(\mathrm{m} / \mathrm{m})\end{array}$ \\
\hline C14:0 & Ácido mirístico & $<0,5$ \\
\hline C16:0 & Ácido palmítico & $7,0-14,0$ \\
\hline C18:0 & Ácido esteárico & $1,4-5,5$ \\
\hline C18:1 & Ácido oleico & $19,0-30,0$ \\
\hline C18:2 & Ácido linoleico & $44,0-62,0$ \\
\hline C18:3 & Ácido linolênico & $4,0-11,0$ \\
\hline
\end{tabular}

Fonte: Agência Nacional de Vigilância Sanitária (2000)

Tabela 1 - Propriedades dos biodieseis metílico e etílico de soja

\begin{tabular}{|c|c|c|c|}
\hline \multirow{2}{*}{ Propriedades } & \multicolumn{2}{|c|}{ Biodiesel } & \multirow{2}{*}{$\begin{array}{l}\text { Especificação para Biodiesel (Reso- } \\
\text { lução nº 14/2012 da ANP) }\end{array}$} \\
\hline & Metílico & Etílico & \\
\hline Índice de Acidez (mg KOH/g) & 0,69 & 0,55 & 0,5 \\
\hline Índice de Iodo $\left(\mathrm{g} \mathrm{I}_{2} / 100 \mathrm{~g}\right)$ & 114 & 105 & Anotar \\
\hline Umidade (\% água) & 0,04 & 0,05 & $*$ \\
\hline Glicerina Livre \% (m/m) & 0,01 & 0,01 & 0,02 \\
\hline Glicerina Total \% $(\mathrm{m} / \mathrm{m})$ & 0,19 & 0,17 & 0,25 \\
\hline Viscosidade Cinemática a $40^{\circ} \mathrm{C}\left(\mathrm{mm}^{2} \cdot \mathrm{s}^{-1}\right)$ & 5,75 & 5,83 & $3,0-6,0$ \\
\hline Massa Específica a $20^{\circ} \mathrm{C}\left(\mathrm{kg} / \mathrm{m}^{3}\right)$ & 882,8 & 878,4 & $850-900$ \\
\hline Ponto de Fulgor, $\min \left({ }^{\circ} \mathrm{C}\right)$ & 168 & 170 & 100 \\
\hline Enxofre Total, máx. (\%) & 0 & 0 & 0,001 \\
\hline Índice de Cetano, mín. & 56 & 60 & Anotar \\
\hline Ponto de Entupimento de Filtro a Frio & -5 & 10 & $* *$ \\
\hline Corrosividade ao Cobre, $3 \mathrm{~h}$ a $50^{\circ} \mathrm{C}$, máx. & 1 & 1 & 1 \\
\hline
\end{tabular}

* O limite máximo de Água e sedimentos é 0,038\% (380 mg.kg-1) até 60 dias depois da data de publicação da Resolução ANP 14/2012. Depois de $1^{0}$ de janeiro de 2013 até 31 de dezembro de 2013, será admitido o limite máximo de 0,035\% (350 mg.kg-1). Depois de 10 de janeiro de 2014, o limite máximo será 0,020\% (200 mg.kg-1); ** O Ponto de Entupimento de Filtro a Frio varia de acordo com a área de comercialização do biocombustível.

Fonte: Adaptada de Candeia (2008) e Agência Nacional do Petróleo, Gás Natural e Biocombustíveis (2012b)

A composição do óleo exerce forte influência sobre várias propriedades do biodiesel diretamente ligadas ao seu desempenho, como massa específica, viscosidade cinemática, número de cetano, ponto de névoa, ponto de entupimento de filtro a frio e ponto de fluidez (LÔBO; FERREIRA; DA CRUZ, 2009). O óleo de soja apresenta um percentual muito alto de ácidos graxos insaturados, e este é um fator determinante na estabilidade oxidativa do biodiesel.

\subsection{Parâmetros de qualidade do biodiesel}

Para evitar danos no motor e assegurar bom desempenho, qualidade nas emissões resultantes da queima e segurança no transporte e manuseio, foram estabelecidos parâmetros de qualidade que determinam limites de contaminantes e especificam as propriedades físico-químicas que o biodiesel deve apresentar. No Brasil, essas especificações são instituídas e monitoradas pela ANP, por meio da Resolução no 14 de 2012 (RANP 14/12) (AGÊNCIA NACIONAL DO PETRÓLEO, GÁS NATURAL E BIOCOMBUSTÍVEIS, 2012b).

Dentre os parâmetros de qualidade do biodiesel há os parâmetros gerais, que também são aplicados ao diesel de petróleo, e há um grupo especial de parâmetros relacionados à composição química e pureza dos óleos vegetais (LÔBO; FERREIRA; DA CRUZ, 2009). 


\subsection{Estabilidade oxidativa e método rancimat}

A oxidação do biodiesel é uma reação que se inicia nas insaturações dos ésteres de ácidos graxos e afeta outras propriedades do biodiesel, como viscosidade cinemática, número de cetanos e índice de acidez (JAIN; SHARMA, 2011). Está relacionada com o grau de instauração e com as posições das duplas ligações (LÔBO; FERREIRA; DA CRUZ, 2009).

Diversos fatores afetam a estabilidade à oxidação do biodiesel, como radiações ultravioleta, umidade, contaminação por metais, alta temperatura e exposição ao ar durante o tempo de estocagem (SARIN et al., 2009), (DANTAS et al., 2011).

Durante a reação, são formados os produtos primários da oxidação, que se decompõem para formar os produtos secundários, por sua vez mais estáveis. Os principais produtos secundários formados são aldeídos, cetonas, hidrocarbonetos, epóxidos, álcoois e ácidos carboxílicos de cadeia curta (KNOTHE, 2007), ( RAMALHO; JORGE, 2006), que corroem as partes metálicas do motor e causam entupimento do filtro de combustível e carbonização no injetor (SARIN et al., 2009).

O acompanhamento da degradação do biodiesel, durante o período de armazenamento, pode ser feito através do monitoramento de alguns parâmetros, como a viscosidade, o índice de peróxido e, sobretudo, o período de indução de Rancimat (LÔBO; FERREIRA; DA CRUZ, 2009).

O método Rancimat serve para avaliar a estabilidade oxidativa do biodiesel através de um teste acelerado de oxidação (LÔBO; FERREIRA; DA CRUZ, 2009). Consiste em um sistema composto por um recipiente reacional ligado a uma célula monitorada por um eletrodo, conforme ilustrado pela Figura 2.

Figura 2 - Teste acelerado de oxidação pelo método Rancimat

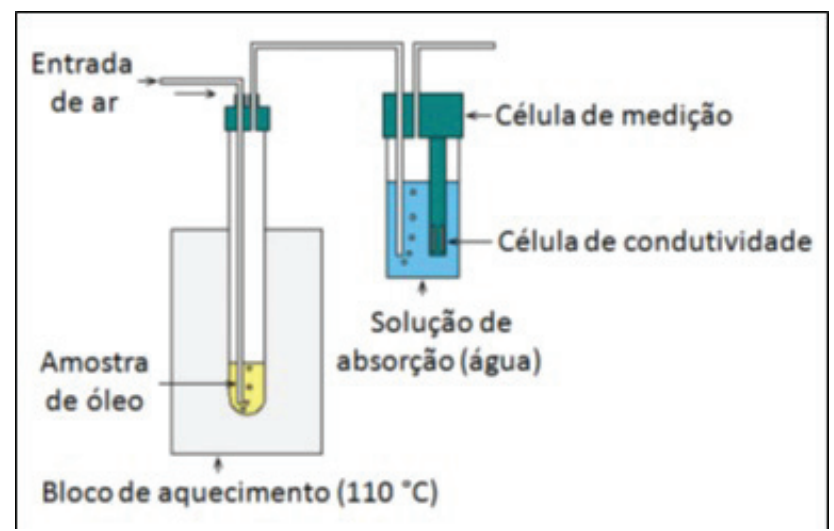

Fonte: Santos (2008)

A amostra é colocada num recipiente reacional, em um bloco de aquecimento a $110^{\circ} \mathrm{C}$, e por ela passa um fluxo contínuo de ar. Com o aumento da temperatura e da quantidade de oxigênio, a oxidação do biodiesel é induzida.
São formados os produtos primários, seguidos dos produtos secundários da oxidação, dentre os quais estão ácidos orgânicos voláteis de cadeia curta. Esses ácidos são carreados para uma célula contendo água deionizada e promovem o aumento da condutividade, que é medida por um eletrodo acoplado a um dispositivo que registra a condutividade em função do tempo (FOCKE et al., 2012), (SANTOS, 2008), (CINI et al., 2012).

O Período de Indução (PI) é o tempo em que a curva de condutividade cresce rapidamente, resultando em uma inflexão, correspondente ao aparecimento dos produtos secundários da oxidação. A RANP 14/12, assim como a Norma EN 14214 do Comitê Europeu de Normalização, estabeleceu o método Rancimat como padrão com um mínimo de $6 \mathrm{~h}$ de período de indução a $110^{\circ} \mathrm{C}$. A Norma ASTM D6751 da American Society of Testing and Materials, que também adotou o método Rancimat, especifica $3 \mathrm{~h}$ de período de indução, no mínimo (LÔBO; FERREIRA; DA CRUZ, 2009), (AGÊNCIA NACIONAL DO PETRÓLEO, GÁS NATURAL E BIOCOMBUSTÍVEIS, 2012b), (FOCKE et al., 2012).

\subsection{Voltametria cíclica}

Os métodos voltamétricos são métodos eletroanalíticos que dependem da medida da corrente em função do potencial aplicado. Baseiam-se na medida da corrente de uma célula eletroquímica sob condições de completa polarização, na qual a velocidade de oxidação ou redução do analito é limitada pela velocidade de transferência de massa do analito para a superfície do eletrodo (SKOOG et al., 2008).

A voltametria cíclica (VC) é uma técnica eletroanalítica importante e amplamente empregada. É frequentemente utilizada no estudo de reações redox, na detecção de intermediários de reação e na observação e no acompanhamento de reações envolvendo produtos formados nos eletrodos (SKOOG et al., 2008).

Em VC, o potencial de um eletrodo de trabalho pequeno e estacionário é variado linearmente com o tempo, começando de um potencial no qual o eletrodo é inerte e seguindo para potenciais nos quais a redução ou oxidação do analito ocorrem. Depois de passar por essa faixa de potencial no qual uma ou mais reações ocorrem, a direção da varredura é invertida e as reações dos intermediários e produtos, formados durante a varredura anterior, frequentemente são detectadas no eletrodo (EVANS et al., 1983).

\subsection{Sensores de ouro}

Sensores de metais nobres, especialmente ouro, têm sido bastante estudados devido às suas propriedades térmicas, óticas e catalíticas, bem como sua atividade eletrônica, e têm recebido diversas aplicações nos campos da física, química, biologia, medicina e ciência de materiais. Para a química eletro- 
analítica, os sensores de ouro têm sido bastante utilizados por causa da sua boa compatibilidade biológica, excelente capacidade condutiva e grande raio superfície/volume (GUO; WANG, 2007).

Eletrodos de ouro em diferentes formas, como eletrodo sólido, impresso ou com filme, tem sido utilizados para detectar metais pesados como chumbo, cobre e mercúrio em análises voltamétricas de redissolução (LASCHI; PALCHETTI; MASCINI, 2006). Além disso, eletrodos de ouro têm apresentado alta atividade catalítica em processos de oxidação eletrolítica de substâncias como, por exemplo, óxido nítrico, hidrazina e metanol (GUO; WANG, 2007).

\section{PROCEDIMENTOS EXPERIMENTAIS}

\subsection{Equipamentos e materiais}

A determinação do Período de Indução do biodiesel de soja foi feita através do equipamento Rancimat da marca Metrohm, modelo 743, do Laboratório de Análises e Pesquisa em Química Analítica de Petróleo e Biocombustíveis (LAPQAP) da Universidade Federal do Maranhão. Utilizou-se o método padronizado pela Norma EM 14112, adotado no Brasil pela ANP.

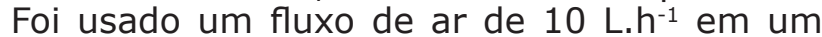
recipiente reacional, no bloco de aquecimento a $110^{\circ} \mathrm{C}$, acoplado à célula de medição abastecida por água deionizada.

Para obtenção dos dados experimentais eletroquímicos, utilizou-se um analisador eletroquímico Autolab da marca Metrohm, modelo PGSTAT 302, acoplado a um computador para registro de dados, do Laboratório de Pesquisa em Química Analítica (LPQA) da UFMA.

Os experimentos eletroquímicos foram con-

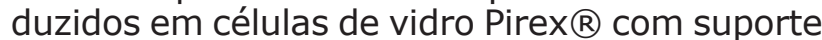
para até $10 \mathrm{~mL}$, usando-se um eletrodo de ouro como eletrodo de trabalho, um eletrodo saturado de $\mathrm{Ag} \mid \mathrm{AgCl}$ com solução de $\mathrm{KCl}$ e um fio de platina como eletrodos de referência e auxiliar, respectivamente. A temperatura durante os experimentos eletroquímicos foi $25 \pm 1^{\circ} \mathrm{C}$.

\subsection{Reagentes e soluções}

Todos os reagentes utilizados foram de grau analítico. A água utilizada foi destilada e posteriormente deionizada a uma resistividade de 18,2 M $\Omega$.cm em um sistema Milli-Q Millipore (Bedford). Na síntese do biodiesel, foi utilizado óleo vegetal de soja comercial (Sadia), metanol P.A. (Merck), hidróxido de potássio (Neon) e ácido clorídrico fumegante 37\% (Merck).

A solução de cloreto de lítio $0,1 \mathrm{~mol}^{-\mathrm{L}^{-1}}$ foi preparada através da diluição de 0,212 g do sal (Vetec) em acetonitrila (Merck), em um balão de $50 \mathrm{~mL}$, o qual ficou no banho de ultrassom por 30 minutos para solubilizar, tendo em vista a difícil solubilidade de cloreto de lítio em meios orgânicos. A solução só pôde ser utilizada após 24 horas, quando a solubilização se completou.
A solução de hidróxido de amônia 0,1 mol.L ${ }^{-1}$, também em meio orgânico, foi preparada a partir da diluição de 0,175 g $(0,688 \mathrm{~mL})$ deste hidróxido (Cromoline) em acetonitrila (Merck), para um volume de $50 \mathrm{~mL}$ de solução.

A solução de ácido nítrico $0,1 \mathrm{~mol} . \mathrm{L}^{-1}$, em meio orgânico, foi preparada diluindo-se 0,315 $\mathrm{g}(0,349 \mathrm{~mL})$ de ácido nítrico (Merck) em acetonitrila (Merck) para um volume de $50 \mathrm{~mL}$, aferido em um balão volumétrico.

As alíquotas necessárias dos reagentes para o preparo dessas soluções, bem como as adições de biodiesel na célula eletroquímica, foram efetuadas com micropipetas Digipet com capacidades nas faixas de 10 a $100 \mu \mathrm{L}$ e 100 a $1000 \mu \mathrm{L}$.

\subsection{Procedimentos e medidas experimentais}

\subsubsection{Síntese do biodiesel de soja}

A síntese do biodiesel foi realizada conforme descrito por Ghisi (GHISI, 2011). Foram utilizados aproximadamente $200 \mathrm{~g}$ de óleo vegetal de soja. Para cada $100 \mathrm{~g}$ do óleo de soja, foram utilizados $35 \mathrm{~mL}$ de metanol e $1,5 \mathrm{~g}$ do catalisador hidróxido de potássio. A produção do biodiesel foi feita no LPQA.

O óleo de soja foi previamente seco em estufa durante 2 horas a $80^{\circ} \mathrm{C}$. Inicialmente foi obtido o metóxido de potássio misturando-se o álcool metílico com o hidróxido de potássio, sob agitação magnética até a homogeneização completa. Adicionou-se ao óleo de soja seco o metóxido de potássio, misturando-se por 2 horas sob agitação constante em agitador mecânico. Ao final da reação, a mistura foi transferida para um funil de decantação, com a finalidade de separar as fases.

Após o repouso de $24 \mathrm{~h}$, observaramse duas fases bem distintas: uma contendo ésteres, clara e menos densa, e outra rica em glicerina, mais densa e escura. A glicerina foi retirada e o biodiesel foi purificado pelo processo de lavagem. O biodiesel foi neutralizado com solução aquosa de $\mathrm{HCl} 0,5 \%$ $\mathrm{v} / \mathrm{v}$ e, em seguida, lavado quatro vezes com água destilada. A cada adição de água, o biodiesel foi deixado em repouso, decantando por 30 minutos para que houvesse a separação das fases aquosa e orgânica. Em seguida, o biodiesel purificado, cuja água retirada depois da lavagem apresentava $\mathrm{pH}$ próximo de 7,0, foi levado para a estufa por aproximadamente 4 horas a $100^{\circ} \mathrm{C}$ para eliminar traços de água e álcool que ainda poderiam estar presentes.

\subsubsection{Avaliação da estabilidade oxidativa} através do método rancimat

Além da presença de metais, fatores como oxigênio, temperatura, luz, entre outros, podem aumentar a oxidação do biodiesel, por isso alguns cuidados foram tomados no armazenamento da amostra a fim de reduzir a influência destes fatores sobre a estabilidade do biodiesel. Assim, após o ensaio no Rancimat, a amostra foi armazenada em frasco vedado com 
Parafilm $®$ e acondicionada em geladeira $(10 \pm$ 3 oC), protegida da luz. O ensaio de estabilidade oxidativa foi executado segundo a Norma EN 14112 . Alíquotas de $3.0 \mathrm{~g}$ de amostra foram pesadas em cada frasco para as análises no Rancimat, sendo estas realizadas em triplicata.

\subsubsection{Avaliação do comportamento eletroquími-} co do sistema em meios salino, básico e ácido

Para cada teste, foram adicionados à célula eletroquímica $10 \mathrm{~mL}$ das soluções do eletrólito de suporte diluído em acetonitrila.

Utilizou-se a solução de cloreto de lítio 0,1 mol.L-1 para o teste em meio salino e foram feitas adições de 20, 40, 60, 80 e $100 \mu \mathrm{L}$ de biodiesel de soja, correspondentes às seguintes concentrações na célula: 1,78, 3,55, 5,33 e 7,11 mg.mL-1.

No teste em meio básico, utilizou-se como eletrólito de suporte a solução de hidróxido de amônio 0,1 mol.L-1 e foram feitas adições de 50,100 e $150 \mu \mathrm{L}$ de biodiesel de soja, correspondentes, respectivamente, a 4,44, 8,89 e 13,33 mg.mL-1 de concentração na célula.

No teste em meio ácido, foram feitas adições de 100, 200, 300, 400 e $500 \mu \mathrm{L}$ de biodiesel de soja em solução de ácido nítrico 0,1 mol.L-1, que correspondem a concentrações de $8,89,17,77,26,66,35,54$ e 44,43 mg.mL-1.

\subsubsection{Otimização das condições experimentais}

Alguns parâmetros, como potencial inicial, potencial de retorno, potencial final e o número de ciclos, foram otimizados antes das análises de biodiesel, a fim de se obter condições ideais para a obtenção da resposta analítica. Desse modo, um parâmetro foi variado por vez, enquanto os demais permaneceram constantes. Para efetuar a otimização, $10 \mathrm{~mL}$ do eletrólito de suporte escolhido (solução de ácido nítrico $0,1 \mathrm{~mol}$ L-1, em acetonitrila) foram adicionados à célula eletroquímica. A Tabela 3 mostra os parâmetros escolhidos após a otimização das melhores condições experimentais estudadas.

Tabela 3 - Parâmetros eletroquímicos para a oxidação de biodiesel por voltametria cíclica

\begin{tabular}{c|c}
\hline Condições Experimentais & Especificações \\
\hline Tempo de Desaeração & $10 \mathrm{~min}$ \\
\hline Tempo de Equilíbrio & $10 \mathrm{~s}$ \\
\hline Potencial Inicial & $-0,1 \mathrm{~V}$ \\
\hline Potencial de Retorno & $+1,5 \mathrm{~V}$ \\
\hline Potencial Final & $-0,1 \mathrm{~V}$ \\
\hline Velocidade de Varredura & $100{\mathrm{mV} . \mathrm{s}^{-1}}^{-10}$ \\
\hline Direção Inicial da Varredura & Anódica \\
\hline Modo de Varredura & Linear \\
\hline Número de Ciclos & 5 \\
\hline Eletrólito de suporte & $\mathrm{HNO}$ \\
\hline
\end{tabular}

Fonte: Elaborada pelos autores
2.3.5 Estudo do comportamento eletroquímico do biodiesel

Depois de otimizados os parâmetros operacionais e as condições experimentais, a amostra de biodiesel de soja foi submetida às medidas voltamétricas cíclicas, conforme 0 seguinte procedimento: foram adicionados 10 $\mathrm{mL}$ do eletrólito de suporte à célula, na qual inseriu-se o eletrodo de ouro limpo, conforme procedimento de limpeza anteriormente citado. Os eletrodos de referência e auxiliar foram lavados com água deionizada, enxutos e introduzidos na célula. Durante 10 minutos, a célula ficou sob agitação magnética e desaeração com gás nitrogênio.

A Tabela 4 apresenta os volumes das adições de biodiesel e suas correspondentes concentrações na célula. Entre cada adição, a célula ficava sob agitação magnética por 5 minutos para a completa solubilização da amostra e o eletrodo de ouro era polido no feltro com água deionizada.

Tabela 4 - Adições de biodiesel para estudo do comportamento eletroquímico em meio ácido

\begin{tabular}{c|c}
\hline Volumes de biodiesel $(\mu \mathrm{L})$ & $\begin{array}{c}\text { Concentrações de biodiesel } \\
\left(\mathrm{mg} \cdot \mathrm{mL}^{-1}\right)\end{array}$ \\
\hline 20 & 1,78 \\
\hline 40 & 3,55 \\
\hline 60 & 5,33 \\
\hline 80 & 7,11 \\
\hline 100 & 8,89 \\
\hline 120 & 10,66 \\
\hline 140 & 12,44 \\
\hline 160 & 14,22 \\
\hline
\end{tabular}

Fonte: Elaborada pelos autores

\section{RESULTADOS E DISCUSSÃO}

\subsection{Estudo da estabilidade oxidativa pelo método Rancimat}

A Norma Europeia EN 14112, adotada pela ANP, que trata do Teste de Estabilidade Oxidativa do Biodiesel, foi usada como referência no presente estudo, através do método de Rancimat. Três amostras de aproximadamente $3,0 \mathrm{~g}$ foram utilizadas no teste. Os valores de período de indução (PI) obtidos estão listados na Tabela 5.

Tabela 5 - Valores de período de indução (PI) obtidos para as amostras do biodiesel metílico de soja

\begin{tabular}{c|c}
\hline Amostras & Período de Indução - PI (h) \\
\hline Amostra A & 4,52 \\
\hline Amostra B & 5,04 \\
\hline Amostra C & 5,09 \\
\hline PI médio & 4,88 \\
\hline
\end{tabular}

Fonte: Elaborada pelos autores 
$O$ valor do PI médio encontrado para o biodiesel de soja foi de $4,88 \mathrm{~h}$, como é mostrado na Tabela 5. Este biodiesel não atingiu o período de indução mínimo de $6 \mathrm{~h}$ descrito pelo método Rancimat e, assim, não se encontra em conformidade com as especificações da ANP, através da RANP 14/12. Porém, o valor encontrado ainda está de acordo com as especificações mínimas de $3 \mathrm{~h}$ de PI, considerando a Norma ASTM D6751 (LÔBO; FERREIRA; DA CCRUZ, 2009), (AGÊNCIA NACIONAL DO PETRÓLEO, GÁS NATURAL E BIOCOMBUSTÍVEIS, 2012b), (FOCKE et al., 2012).

A baixa estabilidade à oxidação do biodiesel metílico de soja, constatada pelo valor de PI igual a $4,88 \mathrm{~h}$, está relacionada com as estruturas dos ácidos graxos que o compõem. O biodiesel conserva boa parte da composição do óleo vegetal que o originou, havendo pequenas perdas durante o processo de transesterificação ou armazenamento (FOCKE et al., 2012), (KNOTHE, 2007). O óleo de soja possui alto teor de ácidos graxos insaturados, como o ácido oleico (com uma instauração em sua molécula), que pode compor de $19 \%$ a $30 \%$ da massa do óleo, e o ácido linoléico (com duas insaturações), que varia de $44 \%$ a $62 \%$ (AGÊNCIA NACIONAL DE VIGILÂNCIA SANITÁRIA, 2000), sendo este último o principal responsável pela rápida degradação oxidativa do biodiesel de soja (FERRARI; OLIVEIRA; SCABIO, 2005).

\subsection{Comportamento eletroquímico do Sistema em diferentes meios: salino, bá- sico e ácido}

Um estudo preliminar foi feito para avaliar o comportamento do eletrodo de ouro com adições de biodiesel em meio orgânico, com o objetivo de comparar o seu desempenho nos meios salino, básico e ácido.

A Figura 3 apresenta o comportamento do sistema em meio salino (solução de cloreto de lítio 0,1 mol. $\mathrm{L}^{-1}$ ) em acetonitrila. Cinco adições de biodiesel foram feitas, correspondentes às concentrações de 1,78 , 3,55, 5,33 e 7,11 mg. $\mathrm{mL}^{-1}$, e, com elas, observou-se o decaimento da corrente, o que pode ser atribuído a um aumento da resistividade no meio. Porém, nas últimas adições, pôde-se notar que houve uma saturação no sistema, dificultando o estudo de concentrações superiores a $5,33 \mathrm{mg} \cdot \mathrm{mL}^{-1}$ de biodiesel.

A Figura 4 apresenta o comportamento voltamétrico do sistema em solução de hidróxido de amônio 0,1 mol. $\mathrm{L}^{-1}$, também em meio orgânico. O sistema não se mostrou sensível para a oxidação da amostra de biodiesel. Após três adições de amostra (4,44, 8,89 e 13,33 $\mathrm{mg} \cdot \mathrm{mL}^{-1}$ ), não se observou qualquer alteração na resposta voltamétrica após a adição de biodiesel. Isto indica claramente que o meio básico avaliado não é adequado.

A Figura 5 exibe o comportamento do sistema em meio orgânico, usando como eletrólito de suporte ácido nítrico 0,1 mol.L $\mathrm{L}^{-1}$, para as concentrações 8,89 , $17,77,26,66,35,54$ e $44,43 \mathrm{mg} \cdot \mathrm{mL}^{-1}$ de biodiesel. Observa-se um crescimento de corrente, sensível, estável e diretamente proporcional às adições de biodiesel, em ampla faixa de adição da amostra, o que não foi observado para os outros meios anteriormente estudados. O meio ácido foi o que apresentou melhor comportamento voltamétrico e maior estabilidade do sinal analítico (corrente), quando comparado com os outros meios estudados, sendo, portanto, escolhido para a continuidade dos estudos de otimização.

Figura 3 - Comportamento do sistema em meio orgânico salino ( $\mathrm{LiCl}$ ). Concentrações de (a) 1,78, (b) 3,55 , (c) 5,33 e (d) 7,11 mg.mL-1 de biodiesel de soja

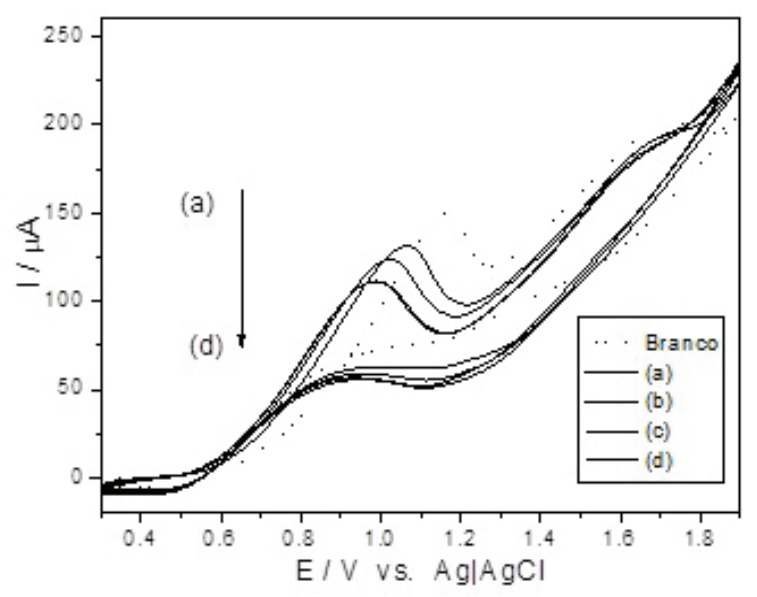

Fonte: Elaborada pelos autores

Figura 4 - Comportamento do sistema em meio orgânico básico $(\mathrm{NH} 4 \mathrm{OH})$. Concentrações de (a) 4,44 , (b) 8,89 e (c) $13,33 \mathrm{mg} \cdot \mathrm{mL}-1$ de biodiesel de soja

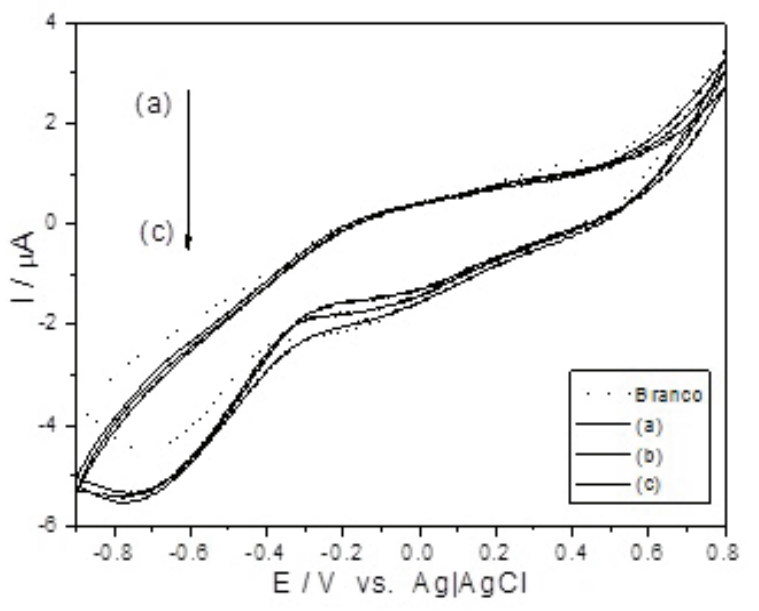

Fonte: Elaborada pelos autores 
Figura 5 - Estudo do comportamento do sistema em meio orgânico ácido (HNO3). Concentração de (a) 8,89 , (b) 17,77 , (c) 26,66 , (d) 35,54 e (e) 44,43 mg.mL-1 de biodiesel de soja

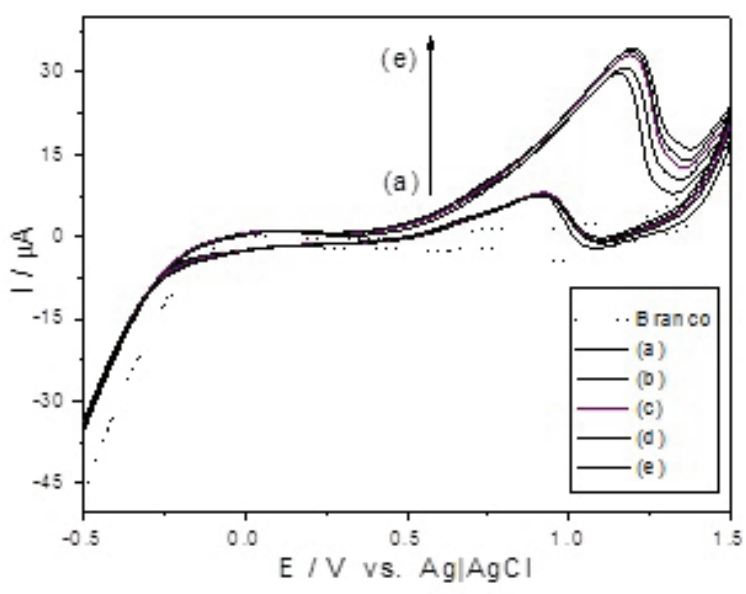

Fonte: Elaborada pelos autores

\subsection{Estudo sobre o comportamento ele- troquímico da oxidação do biodiesel}

O voltamograma da Figura 6 mostra a resposta voltamétrica do sistema em estudo, na ausência [(a): branco] e na presença de Biodiesel $[(b):(1,78 \mathrm{mg} \cdot \mathrm{mL}-1)]$, em ácido nítrico, conforme descrito no capítulo anterior.

Figura 6 - Comparação entre os sinais voltamétricos obtidos na ausência (branco: solução de HNO3 0,1 mol.L-1 em acetonitrila) e presença de biodiesel (1,78 mg.mL-1 do biocombustível na solução/célula)

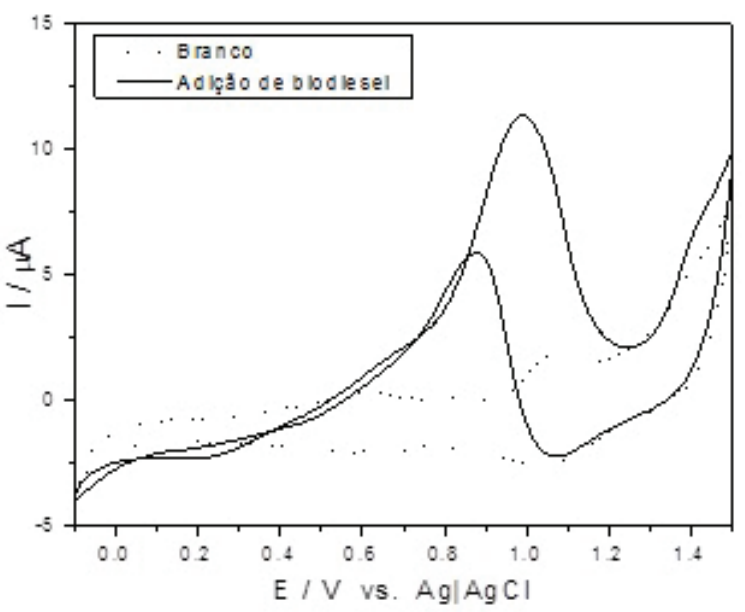

Fonte: Elaborada pelos autores

Observa-se o aparecimento de um processo voltamétrico com dois picos anódicos, sensíveis e bem definidos, próximos a $+0,9 \mathrm{~V}$ e $+1,08 \mathrm{~V}$ (vs. $\mathrm{Ag} \mid \mathrm{AgCl}$ ), sendo que o primeiro corresponde à varredura inversa, no sentido catódico. Tais processos redox podem ser atribuídos à oxidação do Biodiesel. A resposta do branco (a) não apresentou processos redox com correntes significativas nesta região.
Após a adição de mais biodiesel na célula (Figura 7), observou-se o crescimento proporcional da corrente anódica.

Figura 7 - Resposta voltamétrica para a oxidação do biodiesel utilizando voltametria cíclica. Concentração de (a) 1,78, (b) 3,55, (c) 5,33, (d) 7,11, (e) 8,89 , (f) 10,66 , (g) 12,44 e (h) $14,22 \mathrm{mg} \cdot \mathrm{mL}-1 \mathrm{de}$ biodiesel

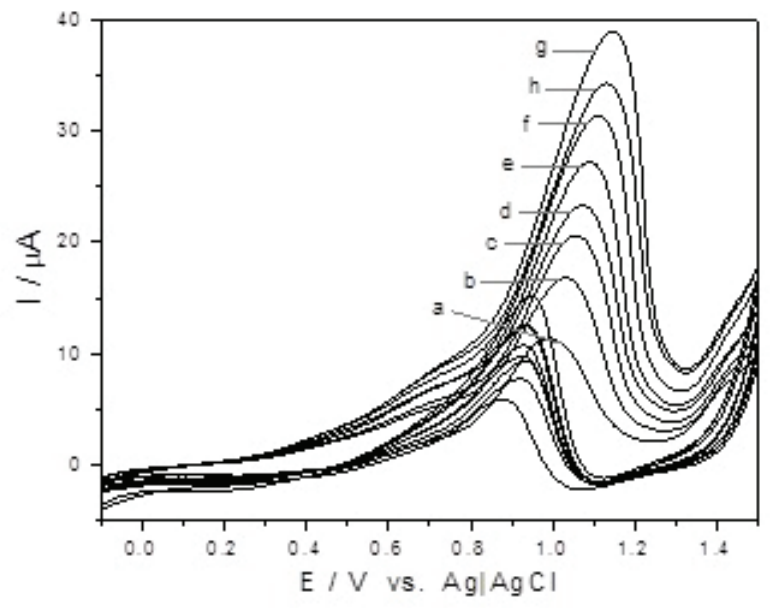

Fonte: Elaborada pelos autores

Com base na resposta voltamétrica observada, uma curva de calibração foi obtida, a qual apresentou uma resposta linear entre a corrente e a concentração de biodiesel, conforme apresentado nas Figuras 8 e 9.

Figura 8 - Curva analítica referente às correntes de pico anódicas em função das concentrações 1,78 , $3,55,5,33,7,11,8.89$ e 10,66 mg.mL-1 de biodiesel

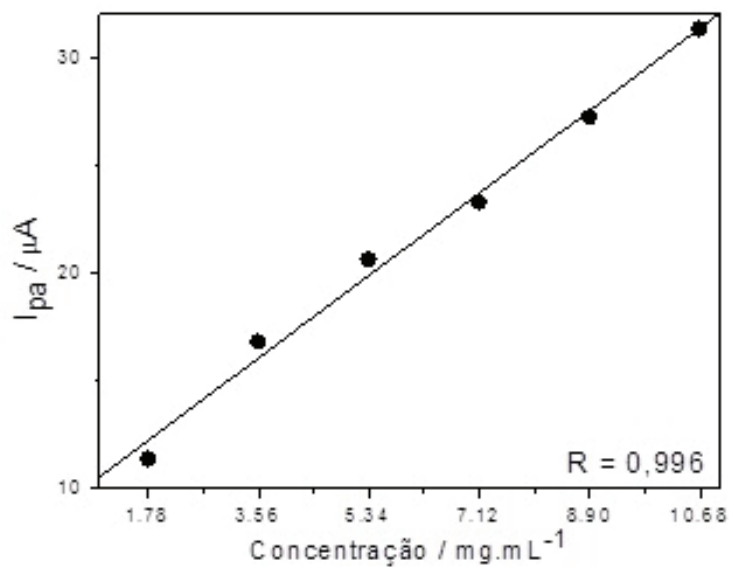

Fonte: Elaborada pelos autores

Uma avaliação da faixa de linearidade é apresentada nas Figuras 8 e 9, onde se observa um excelente coeficiente de correlação $(R=0,996)$ entre 1,78 e 10,66 mg.mL-1 de biodiesel. Observa-se um desvio da linearidade após a concentração de biodiesel correspondente a 10,66 mg.mL1. Quando se inclui a concentração de 12,44 $\mathrm{mg} \cdot \mathrm{mL}-1$, a linearidade cai para $\mathrm{R}=0,990$. 
Figura 9 - Curva analítica referente às correntes de pico anódicas em função das concentrações 1,78 , $3,55,5,33,7,11,8,89,10,66$ e $12,44 \mathrm{mg} \cdot \mathrm{mL}-1$ de biodiesel

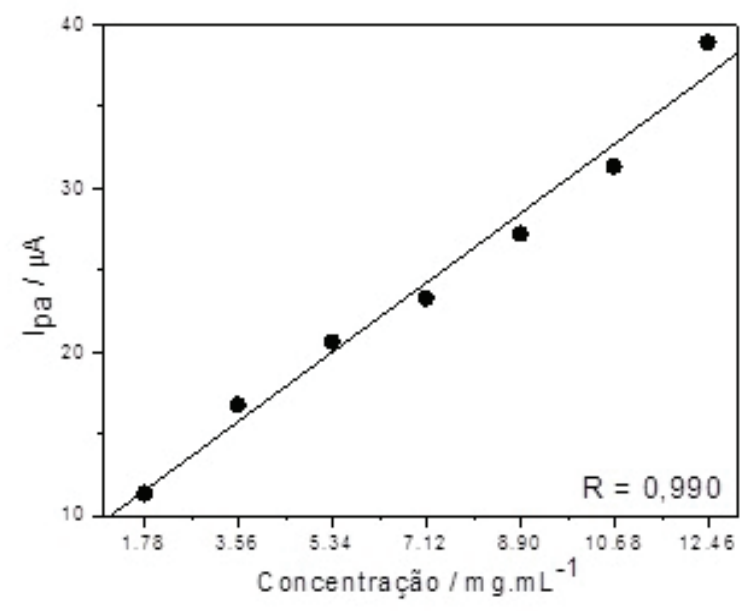

Fonte: Elaborada pelos autores

O desvio da linearidade observado pode ser atribuído à limitação do processo químico na superfície do eletrodo, podendo estar também relacionado com a possível saturação da superfície do eletrodo (CORDEIRO et al., 2006).

Outro fator que pode explicar o decaimento da corrente de pico anódica é o comportamento eletroquímico do solvente utilizado, a acetonitrila $(\mathrm{CH} 3 \mathrm{CN})$. A escolha do solvente deve considerar a solubilidade do eletrólito de suporte e da amostra. Um meio orgânico foi escolhido devido à complexidade da matriz estudada, caso contrário seria necessário fazer um pré-tratamento da amostra, que envolveria ou a formação de uma microemulsão, ou a digestão da amostra, sendo este último inviável para o estudo da oxidação do biodiesel (LUCHO, 2003).

Estudos anteriores mostram que a natureza do meio pode influenciar na resposta de eletrodo de platina, podendo ocorrer uma reação de decomposição da acetonitrila na superfície deste eletrodo. Os resultados desses estudos mostram a influência do potencial do eletrodo, da concentração de água e da competitividade de outras moléculas no processo de adsorção. Outros estudos também apontam que a presença de água favorece a formação da espécie $\mathrm{CH} 3 \mathrm{CONH}$, a qual se adsorve sobre a superfície do eletrodo, na presença de um eletrólito de suporte. Pode ocorrer, com o tempo, a deposição de um filme fino de poliacetonitrila sobre o eletrodo polarizado anodicamente (LUCHO, 2003).

No presente estudo, para evitar a possível formação de filme na superfície do eletrodo, entre cada adição do biodiesel, o eletrodo de ouro era polido e lavado com água deionizada, sendo posteriormente seco, antes de ser novamente introduzido na célula.

Sobre os resultados do presente estudo, vê-se claramente, através das Figuras 8 e 9, que o aumento de corrente, proporcionado pelo aumento da concentração de biodiesel na célula, indica que a resposta eletroquímica é devido à presença de espécie eletroativa no meio, fato este que assegura a viabilidade analítica de um possível procedimento eletroanalítico.

Como é bastante conhecido, devido à dificuldade de se identificar os produtos da complexa reação de oxidação do biodiesel, haverá necessidade de se avaliar diferentes padrões analíticos que possam ser correlacionados com a resposta voltamétrica obtida no presente trabalho, o que identificará as espécies responsáveis pelos processos redox próximos a +0,9 $\mathrm{V}$ e +1,08 $\mathrm{V}$.

\section{CONSIDERAÇÕES FINAIS}

O biodiesel sintetizado apresentou período de indução de 4,88 h, semelhante ao encontrado em trabalhos atualmente desenvolvidos, o que corrobora a sua viabilidade de produção através do método utilizado, para os ensaios que forem realizados futuramente.

Após a otimização do método, optou-se pelo meio orgânico e ácido nítrico como eletrólito de suporte.

A metodologia proposta apresentou boa resposta à oxidação eletroquímica do biodiesel, devido à boa linearidade de crescimento da corrente de pico anódico com as adições da amostra, com coeficiente de correlação 0,996, para concentrações até $10,66 \mathrm{mg} \cdot \mathrm{mL}-1$ de biodiesel na célula. A partir da concentração de $12,44 \mathrm{mg} \cdot \mathrm{mL}-1$, observa-se uma pequena perda de linearidade, que pode ser atribuída à limitação do processo catalítico na superfície do eletrodo de ouro e à possível formação de um filme de poliacetonitrila.

Com os resultados obtidos no presente trabalho, confirma-se a viabilidade do método proposto para estudar eletroquimicamente a oxidação do biodiesel e, como etapa futura, propõe-se a continuidade do estudo visando possível determinação dos intermediários de reação e produtos formados na célula, para melhor caracterização do comportamento oxidativo do biodiesel.

\section{AGRADECIMENTOS}

Os autores agradecem à Fundação de Amparo à Pesquisa e ao Desenvolvimento Científico e Tecnológico do Maranhão (FAPEMA), ao Conselho Nacional de Desenvolvimento Científico e Tecnológico (CNPq), ao Programa de Recursos Humanos da ANP (PRH 39) e à Universidade Federal do Maranhão (UFMA), pelos apoios financeiros e estruturais para a pesquisa e formação de recursos humanos.

\section{REFERÊNCIAS}

\section{AGÊNCIA NACIONAL DE VIGILÂNCIA}

SANITÁRIA. Resolução ANVISA no 482, de

23 de setembro de 1999. Diário Oficial da

União, Brasília, DF, 20 jun. 2000. Disponivel

em: <http://www.anvisa.gov.br/legis/

resol/482_99.htm>. Acesso em: 18 nov. 2012. 
AGÊNCIA NACIONAL DO PETRÓLEO, GÁS NATURAL E BIOCOMBUSTÍVEIS. Anuário estatístico do petróleo, gás natural e biocombustíveis: 2012. Rio de Janeiro, 2012a.

Resolução ANP no 14, de 11 de maio de 2012. Diário Oficial da União, Brasília, DF, 18 maio 2012b. Disponivel em: <http://nxt. anp.gov.br/nxt/gateway.dll/leg/resolucoes anp/2012/maio/ranp\%2014\%20-\%202012. $\mathrm{xml}>$. Acesso em: 18 nov. 2012b.

BALAT, M. Potential alternatives to edible oils for biodiesel production: a review of current work. Energy Conversion and Management, v. 52, p. 1479-1492, 2011.

CANDEIA, R. A. Biodiesel de Soja: síntese, degradação e misturas binárias. 2008. 132f. Tese (Doutorado em Química) - Centro de Ciências Exatas e da Natureza, Universidade Federal da Paraíba, João Pessoa, 2008.

CINI, J. R. D. M. et al. Comparação dos métodos de determinação da estabilidade oxidativa de biodiesel B100, em mistura com antioxidantes sintéticos: aplicação do delineamento simplex-centroide com variável de processo. Química Nova, 2012. Disponivel em: <http://quimicanova.sbq.org.br/qn/ No\%20Prelo/Artigos/AR12344.pdf>. Acesso em: 28 dez. 2012. No prelo.

COLARES, J. F. A brief history of brazilian biofuels legislation. Syracuse Journal of Internacional Law \& Commerce, 35, 2008. 101-116.

CORDEIRO, C. R. B. et al. Ultra trace copper determination by catalytic-adsorptive stripping voltammetry using an alizarin red s modified graphite electrode. International Journal of Electrochemical Science, v. 1, p. 343-353, 2006.

DANTAS, M. B. et al. Evaluation of the oxidative stability of corn biodiesel. Fuel, v. 90, p. 773-778, 2011.

DERMIBAS, A. Biofuels securing the planet's future energy needs. Energy Conversion and Management, v. 50, p. 2239-2249, 2009.

Political, economic and environmental impacts of biofuels: a review. Applied Energy, v. 86, p. 108-117, Nov. 2009.

EVANS, D. H. et al. Cyclic voltammetry. Journal of Chemical Education, v. 60, n. 4, p. 290-293, 1983.

FERRARI, R. A. ; OLIVEIRA, V. D. S.; SCABIO, A. Biodiesel de soja - taxa de conversão em ésteres etílicos, caracterização físico-química e consumo em gerador de energia. Química Nova, v. 28, n. 1, p. 19-23, 2005.

FOCKE, W. W. et al. The effect of synthetic antioxidants on the oxidative stability of biodiesel. Fuel, v. 94, p. 227-233, Apr. 2012.

GHISI, M. Determinação de Cu e Fe em biodiesel por espectrometria de absorção atômica com atomização eletrotérmica e estudo do efeito catalítico destes metais sobre a estabilidade oxidativa do biodiesel. 2011. 91 f. Dissertação (Mestrado em Química) - Universidade Federal do Maranhão, São Luís, 2011.

GUO, S.; WANG, E. Synthesis and electrochemical applications of gold nanoparticles. Analytica Chimica Acta, v. 598, p. 181-192, 2007.

JAIN, S.; SHARMA, M. P. Correlation development for effect of metal contaminants on the oxidation stability of Jatropha curcas biodiesel. Fuel, v. 90, p. 2045-2050, 2011.

KNOTHE, G. Some aspects of biodiesel oxidative stability. Fuel Processing Technology, v. 88, p. 669-677, 2007.

LASCHI, S.; PALCHETTI, I.; MASCINI, M. Gold-based screen-printed sensor for detection of trace lead. Sensors and Actuators $B$, v. 114, p. 460-465, 2006.

LÔBO, I. P.; FERREIRA, S. L. C.; DA CRUZ, R. S. Biodiesel: parâmetros de qualidade e métodos analíticos. Química Nova, v. 32, n. 6, p. 1596-1608, 2009.

LUCHO, A. M. S. Síntese, análise e caracterização do filme polimérico da eletrorredução do furfural sobre platina em acetonitrila. 2003. 103f. Tese (Doutorado em Química) - Instituto de Química, Universidade Federal do Rio Grande do Sul, Porto Alegre, 2003.

MITCHELL, J. F. B.; JOHNS, T. C. On Modification of Global Warming by Sulfate Aerosols. Journal of Climate, v. 10, p. 245267, Feb. 1997.

PRADOS, C. P. et al. Simultaneous gas chromatographic analysis of total esters, mono-, di- and triacylglycerides and free and total glycerol in methyl or ethyl biodiesel. Fuel, v. 96, p. 476-481, June 2012.

RAMALHO, V. C.; JORGE, N. Antioxidantes utilizados em óleos, gorduras e alimentos gordurosos. Química Nova, v. 29, n. 4, p. 755-760, 2006.

SANTOS, J. R. D. J. BIODIESEL DE BABAÇU: avaliação térmica, oxidativa e misturas binárias. João Pessoa: Universidade Federal da Paraíba, p. 103. 2008.

SARIN, A. et al. Influence of metal contaminants on oxidation stability of Jatropha biodiesel. Energy, v. 34, p. 1271-1275, 2009.

SCHUCHARDT, U.; SERCHELI, R.; VARGAS, R. M. Transesterification of Vegetable Oils: a Review. Journal of Brazilian Chemical Society, v. 9, n. 1, p. 199-210, 1998.

SHAHID, E. M.; JAMAL, Y. Production of biodiesel: A technical review. Renewable and Sustainable Energy Reviews, v. 15, p. 4732-4745, 2011.

SKOOG, D. A. et al. Fundamentos de Química Analítica. Trad. Marco Grassi. 8.ed. São Paulo: Cengage Learning, 2008. 